\title{
Microbiome and Metabolome of Patients with Slow Transit Constipation: Unity in Diversity?
}

\author{
Alexandros Hadjivasilis $^{1} \cdot$ Karolos Demetriou $^{2}$ \\ Accepted: 15 October 2020 / Published online: 30 October 2020 \\ (c) Springer Science+Business Media, LLC, part of Springer Nature 2020
}

More than a trillion microorganisms are present in healthy humans, with the majority residing in the lumen of the gastrointestinal (GI) tract, comprising the GI microbiota. Though these microorganisms are mostly bacteria, the microbiota also include viruses, fungi, and protozoa living together and interacting with each other and with the host. A good example of this interaction is termed the gut-brain axis, although the composition of gut microbiota is associated with alterations of the paracellular permeability of the GI tract epithelium [1].

Over the last few years, the gut microbiota have taken center stage by researchers, clinicians, and the public. Many studies now suggest that the microbes in the gut may be involved in the pathogenesis of many gastrointestinal disorders such as inflammatory bowel disease, and irritable bowel syndrome or other functional GI disorders. Interestingly, the gut microbiota may also be involved in extraintestinal diseases such as obesity, the metabolic syndrome, neurological syndromes, cardiovascular diseases, and a host of diverse diseases [2]. A current hypothesis is that gut microorganisms normally live in harmony, impeding the proliferation of pathogenic or other harmful microorganisms, with diseases upsetting this balance [3].

In this issue of Digestive Diseases and Sciences, Tian et al. [4] examined the diversity of gut microbial species and the composition of the gut metabolome in patients with slow transit constipation (STC), a patient population rarely subject to this type of analysis. The authors examined 35 participants in this observational study, 18 with STC meeting the Rome 3 criteria and 17 healthy controls. Microbiota

Alexandros Hadjivasilis

ahadjivasilis@gmail.com

1 Cyprus International Institute for Environmental and Public Health, Cyprus University of Technology, Irinis Str 95, 3041 Limassol, Cyprus

2 School of Medicine, European University of Cyprus, Diogenis Str 6, 2404 Nicosia, Cyprus diversity in fecal samples was analyzed with the standard measures of alpha and beta diversity. The first, a measure of "richness," was significantly greater in STC patients, suggesting that higher microbial diversity is associated with constipation. Moreover, the 2 groups had differences in composition of the microbiome at the family and genus level. Specifically, among others, Bacteroidetes were significantly decreased in STC patients, whereas the number of Firmicutes was increased. Notably, Bifidobacteria abundance was 14.2-fold higher in STC patients than in healthy controls.

The findings of this study raise the question as to whether the altered microbiota in patients with STC are a factor contributing to disease pathogenesis. At the moment, the most supported theory is that decreased microbial richness and diversity correlate with disease [5]. Although there are very few current causal studies, the presence of Bifidobacteria together with Lactobacillus and other related species is considered as beneficial bacteria [1]. Also, Bifidobacteria are one of the main species included in most of the available therapeutic probiotic bacteria preparations [4]. The findings of this study provide some of the first evidence that the composition of the gut microbiota in STC patients more closely resembles that of healthy subjects that that associated with disease. Furthermore, another important question was raised by this study was whether the primary metabolites of the gut microbiota, namely short chain fatty acids (SCFA), contribute to the pathogenesis of STC. Indeed, the authors noted that acetate, the only significantly altered SCFA, was less present in the constipated patients, whereas the other SCFA and total amount were not significantly altered. Given the clear association between acetate and colonic motility [6, 7], this research, combined with published data, suggests that colonic motility can be improved by orally supplements of acetate (vinegar).

Moreover, the abundance of Ruminococcus and disease history were correlated $(\rho=0.519, p=0.027)$ as was the abundance of Roseburia with the concentration of acetate $(\rho=0.606, p=0.018)$ and butyrate $(\rho=0.543, p=0.021)$. 
The increase in Ruminococcus abundance may reflect favorable conditions for fermentative bacteria in STC patients due to slow transit. The decrease in the abundance of Roseburia may affect the host physiological function through the bacterial metabolite SCFA, raising the possibility that this species may be used therapeutically to restore and maintain the balance of the microbiota in STC patients. Last but not least, receiver operating characteristic (ROC) analysis was used to identify any biomarkers of disease, with Prevotella $(\mathrm{AUC}=0.807)$ and acetate $(\mathrm{AUC}=0.758)$ showing the most promise as biomarkers.

The findings of this study can help researchers focus on the distinct microbiota that STC might have and might be different from what was believed until now. STC usually is a chronic disease that affects $15-30 \%$ of patients with constipation. Even though the pathophysiological mechanisms are not clearly understood, it is believed that the disease is mostly due to pathologic alterations of the enteric and the autonomic nervous systems in addition to dysregulation of the neuroendocrine system [8] as is hypothesized with other functional gastrointestinal disorders [1]. Therefore, microbiota-related therapies might worth trying in patients with STC given the caveats that there is no guarantee that orally introduced bacteria will populate the hindgut. Given the dearth of available therapies for the disease, the therapeutic use of probiotic bacteria should be considered.

In conclusion, this important study has shown significant differences between patients with STC and the healthy controls in terms of microbiota composition and diversity. Also, they propose a combination of microbiota species and metabolites to be used as a biomarker for STC. Finally, larger multicenter studies should put more light in this interesting topic.

\section{Compliance with Ethical Standards}

Conflict of interest All authors declare that they have no conflict of interest.

\section{References}

1. Hadjivasilis A, Tsioutis C, Michalinos A, Ntourakis D, Christodoulou DK, Agouridis AP. New insights into irritable bowel syndrome: from pathophysiology to treatment. Ann Gastroenterol. 2019;32:554.

2. Wang B, Yao M, Lv L, Ling Z, Li L. The human microbiota in health and disease. Engineering. 2017;3:71-82.

3. Casén C, Vebø HC, Sekelja M, Hegge FT, Karlsson MK, Ciemniejewska E, et al. Deviations in human gut microbiota: a novel diagnostic test for determining dysbiosis in patients with IBS or IBD. Aliment Pharmacol Ther. 2015;42:71-83.

4. Tian H, Chen, Q, Yang, B, Qin, H, Li, N. Analysis of gut microbiome and metabolite characteristics in patients with slow transit. Dig Dis Sci. (Epub ahead of print). doi: https://doi.org/10.1007/ s10620-020-06500-2.

5. Mosca A, Leclerc M, Hugot JP. Gut microbiota diversity and human diseases: Should we reintroduce key predators in our ecosystem? Front Microbiol. 2016;7:455.

6. Reigstad CS, Salmonson CE, Rainey JF, Szurszewski JH, Linden DR, Sonnenburg JL, et al. Gut microbes promote colonic serotonin production through an effect of short-chain fatty acids on enterochromaffin cells. FASEB J. 2015;29:1395-1403.

7. Vincent AD, Wang XY, Parsons SP, Khan WI, Huizinga JD. Abnormal absorptive colonic motor activity in germ-free mice is rectified by butyrate, an effect possibly mediated by mucosal serotonin. Am J Physiol Gastrointest Liver Physiol. 2018;315:G896-907.

8. Frattini JC, Nogueras JJ. Slow transit constipation: A review of a colonic functional disorder. Clin Colon Rectal Surg. 2008;21:146-152.

Publisher's Note Springer Nature remains neutral with regard to jurisdictional claims in published maps and institutional affiliations. 